\title{
Nouveaux Nématodes \\ du genre Trichospirura Smith et Chitwood, 1967, en Malaisie.
}

\section{Remarques sur l'évolution de la famille des Rhabdochonidae}

\author{
par A.-G. CHABAUD et M. KRISHNASAMY \\ Laboratoire de Zoologie (Vers), associé au C.N.R.S., Muséum national d'Histoire naturelle, \\ 43, rue Cuvier, F 75231 Paris Cedex 05, \\ et Medical Ecology, Institute for Medical Research - Kuala Lumpur - Malaisie
}

\section{Résumé.}

Description de Trichospirura willmotae n. sp., parasite des canaux salivaires de Tupaia glis, et de $T$. sp. (une seule femelle non fécondée), parasite de l'intestin de Myotis mystacinus en Malaisie.

Les deux espèces sont très proches de l'espèce type, parasite des canaux pancréatiques de Primates au Brésil, et s'en distinguent surtout par les dimensions des extrémités postérieures du corps.

Le genre Rhabdochona, parasite de l'intestin des Poissons d'eaux douces, en même temps qu'il subit une évolution morphologique, plus ou moins poussée, mais toujours uniforme, s'adapte à de nombreux hôtes (Poissons de mer, Sauriens, Mammifères) et à de nombreuses localisations. Cette lignée évolutive comprend 6 genres; Trichospirura, le seul qui soit parasite de Mammifère, est morphologiquement l'un des plus évolués.

Quelques remarques sont faites sur le spectre d'hôtes des Trichospirura, sur les rapports entre Rhabdochonidae et Cystidicolidae et sur l'appareil osmo-excréteur des Trichospirura. Cet appareil, dont l'hypertrophie pourrait résulter du passage, au cours de la phylogénie, de la vie aquatique à la vie terrestre, est très comparable à celui des Pneumospiruridae.

\section{Summary.}

New Nematodes of the genus Trichospirura Smith and Chitwood, 1967, in Malaysia. Remarks on the evolution of the family Rhabdochonidae.

Description of Trichospirura willmottae n. sp. parasite of the salivary ducts of Tupaia glis and T. sp. (single virgin female) parasite of the intestine of Myotis mystacinus in Malaysia. 
The two species are very closely related to the type species, a parasite of the pancreatic ducts of brasilian Primates, and can be differentiated mainly by the mensurations of the posterior extremities of the bodies.

While the genus Rhabdochona, parasite of the intestine of fresh-water fishes, underwent a very similar, but more or less pronounced, morphological evolution, it became adapted to many different hosts: Sea-fishes, Saurians, Mammals and to many locations. This evolutionary line includes six genera; Trichospirura, the only parasite in Mammals, is one of the more evolved.

Some remarks are made on the host-distribution of Trichospirura, on the relationships between Rabdochonidae and Cystidicolidae and on the osmo-excretory apparatus of Trichospirura. The hypertrophy of this apparatus, which could be the consequence of the passage during the course of evolution from aquatic to terrestrial life, is comparable to that of the Pneumospirurinae.

\section{I. - Trichospirura willmottae n. sp.}

Matériel: 2 ô, 2 \&, 2 fragments de femelles.

Hôte: Tupaia glis (Diard).

Localisation: canaux des glandes salivaires.

OrIGINE GÉOGRAPHIQUe: Ulu Gombak (proximité de Kuala-Lumpur).

\section{Description.}

Corps très fin en avant, s'élargissant progressivement dans la région œsophagienne, distendu par les œufs en arrière de l'œsophage et sur toute la longueur du corps, puis progressivement atténué à partir d'1 mm environ de l'extrémité postérieure. Ailes latérales absentes. Cuticule très fine portant de fines annelures transversales, assez irrégulières.

Tête très petite (environ $15 \mu$ de diamètre), avec bouche relativement grande, ovalaire, à grand axe allongé dans le sens latéral. Le pourtour buccal, légèrement chitinoïde, porte 4 papilles labiales internes très petites. En arrière de la bouche se distinguent 4 papilles labiales externes, et nettement plus en arrière, 4 papilles céphaliques et 2 amphides.

Pharynx démesurément allongé, avec portion antérieure dilatée en cavité buccale très courte. Esophage musculaire plus court que le pharynx et nettement distinct de l'œsophage glandulaire.

Appareil osmo-régulateur avec canaux dilatés, restant très nettement perceptibles dans les champs latéraux jusqu'à l'extrémité postérieure du corps.

Mâle avec spicules inégaux, le droit court, dilaté en cupule à son apex, le gauche à pointe obtuse. 5 paires de petites papilles postcloacales, la plus postérieure détachée en arrière. 

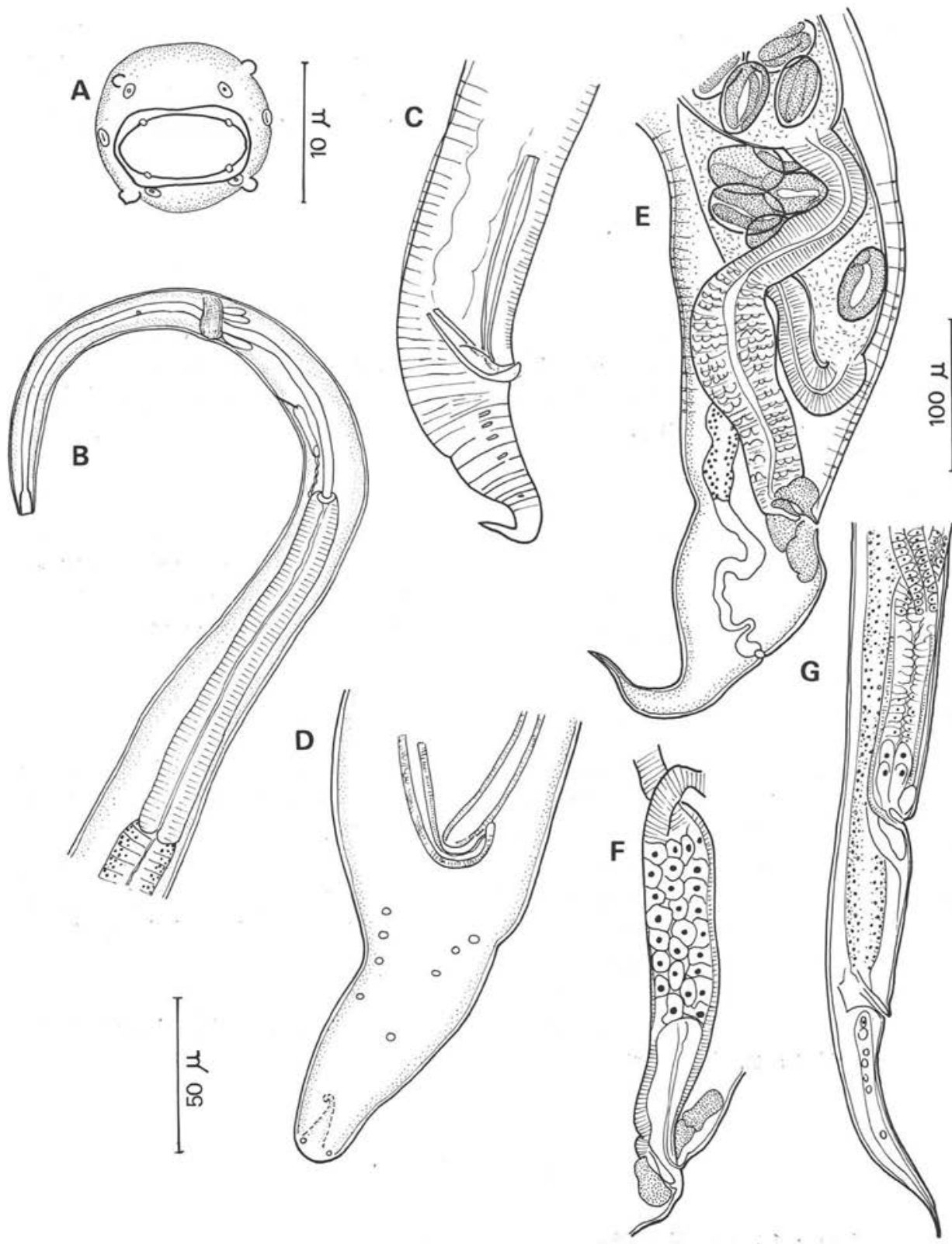

Trichospirura willmottae n. sp. A : extrémité céphalique, $\$$, vue apicale ; B : extrémité antérieure, vue latérale; C: extrémité postérieure, $\hat{\delta}$, vue latérale; $\mathrm{D}: i d$., vue ventrale;

E: extrémité postérieure, $q$ allotype, vue latérale; F : ovéjecteur, autre femelle. Trichospirura sp. G: extrémité postérieure, vue latérale. Echelles : A : $10 \mu ; \mathrm{B}, \mathrm{C}, \mathrm{E}, \mathrm{F}, \mathrm{G}: 100 \mu ; \mathrm{D}: 50 \mu$. 
Femelle avec une vulve très postérieure entourée de formations d'aspect glandu. laire. Ovéjecteur constitué d'un réservoir cylindrique impair dont la portion prévulvaire est obturée par de grandes cellules transparentes, et de 2 branches courtes et musculaires, le reliant à chacun des uteri. Les 2 ovaires sont situés dans la région œsophagienne. Eufs à coque épaisse, embryonnés.

\section{DIMENSIONS DU MÂLE HOLOTYPE.}

Longueur du corps: 7,0 mm. Largeur (sur spécimen non comprimé) au niveau de l'œsophage musculaire: $20 \mu$; au niveau de la partie moyenne du corps : $170 \mu$. Capsule buccale haute de $8 \mu$. Pharynx long de $380 \mu$. Anneau nerveux, deirides, pore excréteur respectivement à $195 \mu, 225 \mu$ et $300 \mu$ de l'apex. CEsophages musculaire et glandulaire longs respectivement de $250 \mu$ et $970 \mu$. Queue longue de $160 \mu$. Spicule droit de $80 \mu$; gauche, $150 \mu$.

\section{Femelle allotype.}

Longueur : 7,5 mm. Largeur sur spécimen non comprimé : $230 \mu$. Pharynx, œsophage musculaire, œsophage glandulaire longs de $340 \mu, 230 \mu$ et $1150 \mu$. Deirides, anneau nerveux et pore excréteur respectivement à $190 \mu, 225 \mu$ et $290 \mu$ de l'apex. Vulve à $240 \mu$ de l'extrémité postérieure. Queue : $140 \mu$. Eufs de $50 \times 32 \mu$.

\section{Discussion.}

Cette espèce a tous les caractères du genre Trichospirura et est très proche de l'espèce type unique du genre: Trichospirura leptostoma Smith et Chitwood, 1967, parasite des canaux pancréatiques de Primates américains.

L'espèce parasite des canaux salivaires du Tupaia peut être séparée par la forme de l'extrémité postérieure de la femelle, plus courte, plus brusquement dilatée et ayant une distance anus-vulve plus réduite. Par ailleurs, le spicule gauche, relativement plus court, a une forme générale moins concave en avant.

Nous estimons donc que le Nématode du Tupaia est nouveau et le nommons Trichospirura willmottae $\mathrm{n}$. sp., en hommage au $\mathrm{D}^{\mathrm{r}}$ Sheila Willmott, directrice d'Helminthological Abstracts.

\section{II. - Trichospirura sp.}

MATÉRIEL: 1 \% non fécondée.

HôTe: Myotis mystacinus (Kuhl).

LOCALISATION : intestin.

ORIGINE GÉOGRAPHIQUe: Bukit Lagong - Kepoog. 16-11-1964. 


\section{Description.}

Corps long de 5,6 mm, large au maximum de $70 \mu$. Pharynx : $340 \mu$. Esophage musculaire : $155 \mu$, glandulaire : $640 \mu$. Deirides, anneau nerveux, pore excréteur respectivement à $180 \mu, 230 \mu$ et $290 \mu$ de l'apex. Queue longue de $170 \mu$; vulve à $280 \mu$ de l'extrémité postérieure.

Ovéjecteur impair long de $130 \mu$, raccordé aux uteri par deux conduits musculeux long de $250 \mu$ et de $420 \mu$. Prodelphie; deux ovaires enroulés dans la région postœesophagienne. Uteri contenant des coques d'œufs et des débris cellulaires.

\section{Discussion.}

Le corps, n'étant pas distendu par les œufs, a un aspect très différent des 2 espèces connues de Trichospirura, mais les différences sont en réalité minimes. La partie paire de l'ovéjecteur est beaucoup plus allongée que chez $T$. willmottae. Ce caractère n'étant pas connu chez $T$. leptostoma, et se modifiant peut-être lorsque les utérus sont distendus, il n'est pas possible d'en tenir compte. Cependant, pour une taille un peu plus faible, les œsophages musculaires et glandulaires sont beaucoup plus courts, alors que la queue et la distance vulve-anus sont plus grandes.

Par les dimensions relatives des organes, cette espèce est donc certainement différente des deux espèces déjà décrites. Ne connaissant que la femelle non fécondée, nous la désignons comme Trichospirura sp.

\section{III. - Place systématique des Trichospirura}

Smith et Chitwood, bien qu'ils comparent à juste titre leur nouveau genre à Vasorhabdochona Martin et Zam, 1967, le placent dans les Thelazioidea, sans vouloir le classer dans une famille déterminée.

A notre avis, au contraire, le genre doit être classé dans les Rhabdochonidae.

Un des éléments les plus caractéristiques dans l'évolution des Nématodes nous paraît être le phénomène de capture : dans de nombreux cas, une lignée de Nématodes, caractéristique d'un groupe d'hôtes déterminés, est capable de s'adapter à des hôtes très différents (fréquemment avec une localisation anormale dans l'organisme) et, en même temps, cette lignée subit une hyperévolution morphologique marquée (Chabaud et Durette-Desset, 1975).

Le genre Trichospirura paraît constituer un bel exemple de ce phénomène de capture :

Nous connaissons, en effet, le genre Rhabdochona Railliet, 1916, groupe de base, très riche en espèces, caractéristique de l'intestin des Poissons d'eaux douces, et toute une série de genres qui s'adaptent (en quittant le plus souvent la localisation intestinale) aux Poissons marins, aux Sauriens et aux Mammifères. 
L'évolution morphologique corrélative à ce phénomène est marquée: par la perte des papilles cloacales et surtout des précloacales, par la perte de la dentition céphalique, puis l'atrophie de la capsule buccale elle-même, par l'allongement du pharynx, par une migration de la vulve vers l'avant ou l'arrière du corps jusqu'à l'acquisition de la monodelphie.

Six genres : Johnstonmawsonia Campana-Rouget, 1955 (= Prosungulonema Roytman, 1963), Freitasia Barus et Coy Otero, 1968, Vasorhabdochona Martin et Zam, 1967, Heptochona Rasheed, 1965 (= Pontochona Mamaev, 1968), Hepatinema Rasheed, 1964, et Trichospirura Smith et Chitwood, 1967, subissent une évolution réalisant de façon plus ou moins poussée le processus morphologique décrit plus haut. Ils appartiennent donc à une lignée homogène, constituée par les Rhabdochona d'eaux douces s'adaptant à de nouveaux habitats (Chabaud, 1975). Trichospirura, en-dehors du fait qu'il est encore didelphe, constitue l'étape morphologique la plus avancée actuellement connue dans cette évolution; c'est le seul qui soit adapté aux Mammifères.

\section{IV. - Remarques sur le genre Trichospirura}

\section{a) Spectre d'hôtes.}

Le genre est maintenant connu dans les canaux pancréatiques de Primates au Brésil, dans les canaux salivaires de Tupaiidae en Malaisie et dans l'intestin de Microchiroptères en Malaisie. Il est possible que cette dernière localisation soit accidentelle, puisque une seule femelle a été récoltée. Peut-être cette troisième espèce est-elle parasite dans les canaux d'une glande acineuse de Chiroptères et échappe ainsi aux recherches.

Les affinités entre parasites de Primates et de Tupaia ne sont pas surprenantes, mais celles avec les parasites de Chiroptères viennent à l'appui des hypothèses formulées sur la parenté phylétique Tupaiidae-Chiroptères suggérée par l'étude des Trichostrongylidae (Chabaud et Durette-Desset, 1975).

\section{b) Relations Rhabdochonidae-Cystidicolidae.}

L'évolution morphologique des Rhabdochona s'adaptant à des hôtes et des habitats divers est, comme il a été démontré plus haut, extrêmement monotone et homogène. Le pharynx, caractéristique, se conserve ou même s'allonge démesurément. Les papilles précloacales s'atrophient. Au contraire, les 4 papilles labiales externes et les 4 céphaliques sont conservées, même à l'étape ultime constituée par le genre Trichospirura.

Les Cystidicolidae qui, eux aussi, s'adaptent à la vie tissulaire, évoluent essentiellement par perte progressive des pseudo-lèvres. Les papilles labiales externes disparaissent rapidement, les papilles cloacales, au contraire, conservent un type Spiruride bien stable. 
Il n'y a donc, ni dans l'origine, ni dans le mode d'évolution, aucun trait commun entre les Rhabdochonidae (Thelazioidea) et les Cystidicolidae (Habronematoidea); le rapprochement des deux groupes qui est fait dans la plupart des travaux récents nous paraît donc être une erreur.

\section{c) Canaux osmo-excréteurs.}

Smith et Chitwood ont figuré des canaux osmo-excréteurs très développés dans les champs latéraux chez l'espèce type. Nous retrouvons cette hypertrophie remarquable chez nos espèces, où les canaux restent aisément perceptibles jusqu'à l'extrémité postérieure du ver.

Le seul groupe de Nématodes où nous connaissions une structure comparable est celui des Pneumospiruridae, qui groupe les Thelazioidea adaptés à la vie pulmonaire chez les Mammifères.

Comme dans le cas des Ascarides, où il y a, au cours de la phylogénie, passage de la vie aquatique à la vie terrestre, on peut supposer que les Nématodes ont besoin de développer un appareil osmo-régulateur plus puissant dans le milieu terrestre, où les variations de pression osmotique au cours du cycle évolutif sont très grandes.

Chez les Ascarides, nous avons supposé que cette adaptation était réalisée par une migration du pore excréteur vers la région céphalique (Chabaud et coll., 1967).

Chez les Thelazioidea, ce pourrait être une hypertrophie simple de l'appareil existant, et, si cette hypothèse était exacte, il faudrait chercher une origine aquatique à l'énigmatique famille des Pneumospiruridae.

\section{Bibliographie}

Barus (V.) et Coy Otero (A.), 1968. - Freitasia teixeirai gen. n. et sp.n. and other Nematodes parasitizing Anolis equestris (Squamata: Iguanidae). Folia Parasit., $15,41-54$.

Campana-Rouget (Y.), 1955. - Sur deux nouveaux genres de Spirurides parasites de Poissons; discussion systématique des genres voisins. Ann. Parasit. hum. comp., 30, 346-362.

Chabaud (A.-G.), 1975. - Keys to genera of the Order Spirurida. Part 1: Camallanoidea, Dracunculoidea, Gnathostomatoidea, Physalopteroidea, Rictularioidea and Thelazioidea. C.I.H. Keys to the Nematode Parasites of Vertebrates $\mathrm{n}^{\circ}$ 3. (Anderson, Chabaud et Willmott éd.), St-Albans, Angleterre, 27 p.

Chabaud (A.-G.), Bain (O.) et Tcheprakoff (R.), 1967. - Redescription de Galeiceps cucullus (Linstow, 1899) et remarques sur l'osmo-régulation des Nématodes Anisakides. Ann. Parasit. hum. comp., 42, 321-326.

Chabaud (A.-G.) et Durette-Desset (M.-C.), 1975. - Remarques sur des Nématodes Trichostrongyloidea qui pourraient indiquer une parenté phylétique entre Tupaiidae et Chiroptères. C.R. Acad. Sc. Paris, 280, sér. D, 201-203. 
MarTin (W.-E.) et ZAM (S.-G.), 1967. - Vasorhabdochona cablei, gen. et sp. n. (Nematoda) from blood vessels of the marine fish, Gillichthys mirabilis Cooper. J. Parasit., 53, 389-391.

RASHEED (S.), 1964. - On Hepatinema karachiensis gen. et sp. nov. (Thelaziidae Railliet, 1916) from a Marine Fish of Pakistan, with a Note on the Genus Filochona (Saidov, 1954) Yamaguti, 1961. J. Helminth., 38, 63-76.

RASHEED (S.), 1965. - A preliminary review of the genus Rhabdochona Railliet, 1916, with a description of a new and related genus. Acta Parasit. Polonica, 13, 407-424.

SмIтH (W. N.) et CHITwood (M. B.), 1967. -- Trichospirura leptostoma gen. et sp. $\mathrm{n}$. (Nematoda: Thelazioidea) from the pancreatic ducts of the white-eared Marmoset Callithrix jacchus. J. Parasit., 53, 1270-1272. 